Its advantage is that it is the time of the morning weather chart, and hence the results can be plotted on the chart with the knowledge that all the observations shown are simultaneous. But the objections are twofold. In many cases figures obtained at great expense have to be rejected, because they are obviously falsified by solar radiation. This must happen if the balloon does not burst, and the sun is high, whereas if the sun is near to or below the horizon it is of no consequence if the balloon does or does not burst. This gives cause for doubt also about some of the printed figures since it is not too clear in all cases as to what may be accepted and what must be rejected. Secondly, it is impossible to get the annual or the daily variation from observations at a fixed hour. The daily variation is, of course, hopeless, and not knowing the law of the daily variation, it is uncertain whether the same correction for the hour should be applied both summer and winter. The result is that the annual variation. above $12 \mathrm{~km}$., as shown by the English ascents, many of which were made at sunset before the international time of 7 a.m. was fixed, differs by $3^{\circ} \mathrm{C}$. from the Continental value, but it is very improbable that there is any real difference. A plan has now been adopted by which the string carrying the instrument uncoils after the balloon is started, and since last winter a very much longer string has been employed in the English ascents. This avoids the difficulty of starting with a long string in rough weather, and it will bc interesting to see what effect the plan will have on the records. The change of length is from 44 to $\mathrm{I}_{32} \mathrm{ft}$.

May 20. W. H. DINES.

\section{Transmission of Electric Waves Round the Bend of the Earth.}

IN a paper on the transmission of electric waves round the earth's surface, read by Prof. H. M. Macdonald before the Royal Society on February 12, some conclusions are recorded which cast new light on the problem of long-distance wireless telegraphy. Prof. Macdonald's point of view is that of simple diffraction. and the paper is the latest one of a notable series of attempts by a number of eminent mathematicians. In the present paper the author reduces his formulæ to figures, and thus makes comparison with experiment easy. The most extensive quantitative experiments yet made over great ranges are those of $\mathrm{L}$. W, Austin in rgro (Bulletin Bureau of Standards, vol. vii., No. 3), and those of J. L. Hogan in I9I3 (Electrician, August 8 , 1913). From the former experiments Austin and $L$. Cohen deduced a formula which has been corroborated by Hogan's results. This formula may be written :-

$$
i=c \epsilon-a \cdot x / \sqrt{\lambda} /(\lambda x),
$$

where $i$ is the current in amperes in the receiving antenna at the distance $x$ kilometres for the sending station, $\lambda$ is the wave-length of the radiation in kilometres, $a$ has the value $0.00 I_{5}$, and $c$, like $a$, is a quantity which does not depend on $\lambda$ or $x$. This formula was deduced from daylight experiments $e x-$ tending over larger ranges of $\lambda$ and $x$ than those used in the table below.

By aid of this formula Prof. Macdonaid's calculations can be quickly compared with the results of experiment. In the following table the first column contains the number of miles between sender and receiver, and the remaining columns contain the ratios of the effect at various distances to that at $4 \mathrm{~J} 9$ miles. $R_{d}$ is the ratio, calculated on the diffraction theory, between the electric fields, $R_{m}$ is the ratio found by measurement between.the currents in the same re- ceiving antenna when moved to the successive distances in turn.

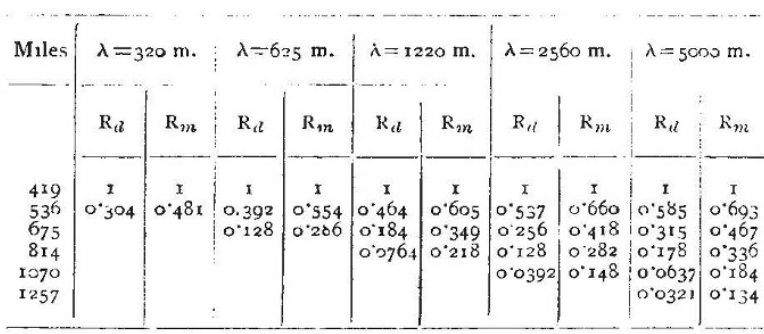

From the table it seems fair to draw the conclusion that diffraction accounts for a large proportion of the observed effects up to distances of, perhaps, 2000 miles. The proportion is much larger than has hitherto been demonstrated, and compels the admission of diffraction into the list of phenomena contributing to the practical success of wireless telegraphy.

But the Austin-Cohen formula expresses a remarkable experimental fact which is not explained, but, rather, is contradicted by the diffraction theory; namely, that for each distance there is a best wavelength. The formula indicates that this optinum wave-length is given by $4 \lambda=a^{2} x^{2}$, and, consequently, that under the best condition

$$
i={ }_{4} c(a \epsilon)^{-2} x^{-3}=c x^{-3} \times 2 \cdot 140 \times 10^{5},
$$

for rather long ranges. These equations are, broadly, borne out by the experience of wireless telegraph engineers. The equation for $\lambda$ shows that as the wave-length is increased the effect at a given place first increases and then decreases. Here the theory of diffraction appears to fail, for the diffraction effect at any fixed point should increase steadily with increase of wave-length. On the other hand, the hypothesis of the refraction of electric waves in the atmosphere when it is ionised by sunlight seems more promising. For while radiation of very short wavelength is lost into space by the rays suffering too little bending, radiation of great wave-length, by being too strongly refracted, is lost in the ground between the oscillator and the receiver; and thus an optimum wave-length is easily conceivable.

University of London, University College, May i8.

\section{Some Phenomena of Clay Suspensions.}

The interesting letters in Nature on the cellular structure of emulsions induced me to test the behaviour of clay which I have been accumulating for some time for evaporation experiments. The clay is obtained by the usual sedimentation method, and is that fraction of the soil which does not settle in twenty-four hours in a beaker containing dilute ammonia to a depth of $8.5 \mathrm{~cm}$. The suspension is then evaporated to dryness in vacuo.

If some of this dried clay be well shaken up with strong ammonia solution and poured into a Petri dish, the usual network, mentioned by Mr. Wager in NATuRE for May 7, gradually develops. Occasionally a different pattern appears, only the angles of the network are formed, and the surface thus has a pitted appearance.

The pattern persists for a few minutes and then gradually becomes blurred. The two cases are shown in Figs. I and 2 respectively. In neither case are groups of cells formed. Although the structure is quite sharp to the eye, the lack of contrast makes the photographic difficulties considerable. I am in.

NO. 2326 , VOL. 93] 